\title{
A Novel Technique for Finding Overlapping Communities in Internet of Things
}

\author{
Jasmine \\ Student (M.Tech) \\ Department of Computer Science, GNDU \\ Regional Campus, Gurdaspur, punjab, INDIA \\ angel.bawa@gmail.com
}

\begin{abstract}
In this paper, we present A Novel Technique for Finding Overlapping Communities in Internet of Things Two concepts named the maximal sub-graph and the clustering coefficient between two neighboring communities are used. In this paper, we have focused on enhancing the Internet of things network further by using the complex networks, complex networks will divide internet of things of users among communities to enhance the services further.
\end{abstract}

Keywords: Internet of Things, Overlapping communities, Clustering Coefficents, Maximal Subgraphs

\section{Introduction}

The Internet of Things (IOT) is a set-up in which objects, people or animals are provided with unique identifiers and the capability to transfer data over a network without the requirement of human-to-human or human-to-computer interaction. IOT has evolved from the junction of wireless technologies, micro-electromechanical systems (MEMS) and the Internet. The Internet of Things (IOT) is a computing perception that explains a future where every day physical objects are associated to the Internet and also capable to categorize themselves to other devices. The term is strongly recognized with Radiofrequency identification (RFID) as the concept of communication, although it also may consist of other sensor technologies, QR codes or wireless technologies.

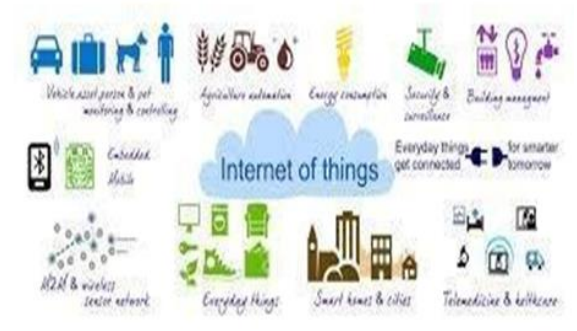

Figure 1. Internet of Things

The Internet of Things (IOT) is the interrelation of uniquely identifiable computing devices within the accessible Internet infrastructure. Many researchers have explained this concept, but Kevin Ashton, an expert on digital improvement was first to work on it. Each definition shares the concept that the first version of it was regarding data created by people, while the next version is regarding data created by things. IoT describe a world where anything can be connected anything at anywhere in an intelligent fashion. 
1.2. Community Structure: One of the most major sub-domains in complex network is the study of community detection. Community detection is vital for many reasons, including node categorization which entails homogeneous groups, group leaders or vital group connectors. Communities may relate to groups of pages of the World Wide Web dealing with correlated topics, to functional modules such as cycles and pathways in metabolic networks, to groups of correlated individuals in social networks etc. The goal is to detect how nodes in the graph will be grouped into communities. Community detection can also been quite useful to derive features for categorization tasks where the data includes connections that are not easily adaptable into features. In various real world networks, communities can split one or more common nodes. For example, in social networks actors may be component of different communities: work, family, friends, and so on. All these communities will distribute a frequent member, and usually more since a work colleague can also be a friend outside the effective environment.

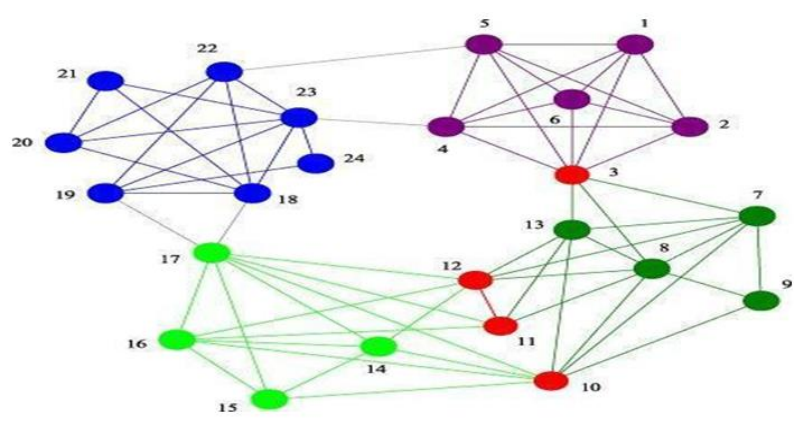

Figure 2. Overlapping Community Structure

In above example, nodes 3,10,11,12 are overlapping nodes i.e. belongs to more than one community. Naturally speaking, a community is a set of nodes that are compactly interrelated but loosely interconnected with the rest of the network. Nodes frequently belong to more than one community i.e. communities can overlap to each other. For example, a person in a social network belongs to many groups, such as a company, a family and a football club; many proteins contain more than one function and belong to multiple functional communities.

2. Literature Survey: Literature is reviewed with respect to detection of overlapping communities and internet of things.

A. Yaozu Cui et al. (2014) presented an alternate algorithm for detecting overlapping community structures in the complex network. Two concepts named the maximal subgraph and the clustering coefficient between two neighboring communities are introduced [1].

B. Laizhong Cui et al. (2014) purposed a weighted modularity based algorithm on the density and

Cohesion as the new evaluation measurement, he proposed three test conditions for overlapping nodes and describes a fast overlapping community detection algorithm with self-correcting ability, which is decaying into two processes [2].

C. Amblard, F. et al. (2010) explained Community detection on networks as a distinguished problem encountered in many fields, for which the present algorithms are ineffective because they do not capture overlapping communities and also in discovering communities containing disparity in size and density, Moreover they do not take into account the networks' dynamics [3].

D. Junqiu Li et al. (2013) said that community detection in the weighted complex networks is useful in understanding the network structures and in the study of the network properties. He presented a unique algorithm to find overlapping communities in the 
weighted complex networks with significant accuracy [4].

E. Alexandre Santos et al. (2014) explained Internet of Things united with Radio Frequency IDentification technology facilitating a complete new context for smart objects that are capable to unite their physical and virtual existences [5].

F.Reza Badie et al. (2013) proposed a novel algorithm that is capable to find overlapping and non-overlapping community structures in complex networks. It uses various agents for analysis of the input network. These agents believe different nodes' closeness in their analysis [6].

G. Hui Suo et al.(2012) explained internet of things (IoT) as a focus of research. Security and privacy are the key tools for IoT applications, and still face some huge challenges. In order to assist this promising domain, he in brief reviews the research progress of IoT, and pay contribution to the security[7].

H. Junsheng Zhang et al.(2013) explains the Internet of Things (IoT) by contributing us a capable and enthralling vision by combining the physical space of smart objects, the virtual cyberspace of information, and society of human effortlessly[8].

\section{Proposed Technique}

Many real-world systems in nature and society can be explained as complex networks or graphs. The entities of the system are described by the nodes (vertices) and the connections between the entities are described by the edges. Examples consist of social interactions, scattering of viruses and diseases, Internet, the World Wide Web, etc. The study of complex networks is significant to be aware of the structure of the networks and the relationships of the entities in the networks. In the past ten years, numerous features about the complex networks could be found from researching the complex networks. One frequent feature is the community structure, where the nodes within a community are highly connected to each other than the nodes among communities. The discovery of community structures has involved much awareness from diverse real networks. Various methods have been planned to discover the community structures of complex networks and implemented successfully to several real complex networks. In this paper, we have focused on enhancing the Internet of things network further by using the complex networks. Complex networks will divide internet of things of users among communities to enhance the services further. The underlying community structure of real-world networks has created scientific and business value. Although many algorithms exist dealing with the problem of community detection it is recognized that the methods involved have a long way to go leaving significant space for improvement.

ACC algorithm [1]

ACC algorithm worked on the clustering coefficient of two neighboring maximal subgraphs can be summarized as follows:

(a) Primarily, use the algorithm to pull out all the maximal sub-graphs from given complex networks with the new tag

(b) Then, compute the number of edges in every maximal sub-graph;

(c) Discover all neighbors of each maximal sub-graph, and compute the clustering coefficient $[\mathrm{Cc}]$;

(d) Take a threshold value $\delta$ whether to combine two communities into a new community and to find the overlapping vertices. 
(e) Repeat steps (c) and (d);

(f) Suspend the repetition and get a proper community structure.

There are so many limitations of earlier techniques due to which the use of complex networks has been ignored for internet of things. Due to hot and recent issue it is required to enhance the internet of things by developing the communities. The effect of energy consumption for internet of things is required to be evaluated, as energy consumption is very essential in today's world. Due to limitations of earlier techniques, we determined to view the problem. Our objectives are simple to evaluate the effect of detecting overlapping communities in networks using the maximal sub-graph and the clustering coefficient and then enhance the internet of things using the maximal sub-graph and the clustering coefficient. For this purpose, we would design simulation environment for internet of things. We will used four metrics to evaluate the performance of internet of things are as follows:

1. Execution time

3. Overhead Time

2. Energy Consumption

4. End To End Delay

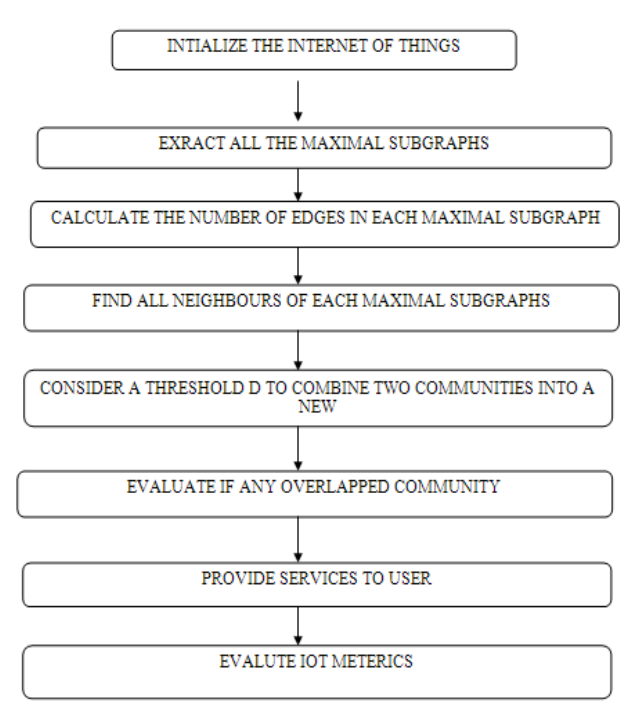

\section{Conclusion}

We proposed a novel technique for detecting the overlapping community structures in internet of things. In this technique, all the maximal sub-graphs first extract and then merge with neighboring maximal sub-graphs to obtain the communities by considering a threshold value. Complex networks will divide internet of things of users among communities to enhance the services further.

\section{References}

[1] Y. Cui, X. Wang and J. Li, "Detecting overlapping communities in networks using the maximal subgraph and the clustering coefficient", Physica A, vol. 405, (2014), pp. 85-91.

[2] L. Cui, L. Qin and N. Lu, "A Fast Overlapping Community Detection Algorithm with Self-Correcting Ability”, (2014) March 13.

[3] R. Cazabet, F. Amblard and C. Hanachi, "Detection of Overlapping Communities in Dynamical Social Networks", (2010) August 20-22.

[4] J. Li, X. Wang and J. Eustace, "Detecting overlapping communities by seed community in weighted complex networks", (2013) December 1.

[5] A. Santos, J. Macedo, A. Costa and M. J. Nicolau, "Internet of Things and Smart Objects for Mhealth Monitoring and Control", vol. 16, (2014). 
[6] R. Badie, A. Aleahmad, M. Asadpour, and M. Rahgozar, "An efficient agent-based algorithm for overlapping community detection using nodes' closeness", vol. 392, Issue 20, (2013), pp. 5231-5247.

[7] H. Suo, J. Wan, C. Zou and J. Liu, "Security in the Internet of Things: A Review", 978-1-4673-0689-8, (2012) March 23-25.

[8] J. Zhang and Y. Sun, "Managing Resources in Internet of Things with Semantic Hyper-Network Model", 978-1-4673-1888-4, (2013) June 27.

[9] M. Coscia, F. Giannotti and D. Pedreschi1, 1Computer Science Department, University of Pisa, Pisa, Italy , 2 KDDLab, ISTI-CNR, Pisa, Italy, 3Center for Complex Network Research, Northeastern University, Boston, USA), “A Classification for Community Discovery Methods in Complex Networks”, (2011).

[10] W. Zhang, G. Pan, Z. Wu and S. Li, (Department of Computer Science, Zhejiang University, China), "Online Community Detection for Large Complex Networks".

[11] G. K. Orman, V. Labatut and H. Cherifi, (Galatasaray University, University of Burgundy), "On accuracy of community discovery algorithms".

[12] L. Atzori, A. Iera and G. Morabito, "The Internet of Things, A survey", (2010), pp. 2787-2805.

[13] H. -I. Wang, "Constructing the Green Campus within the Internet of Things Architecture", (2014) March 5.

[14] A. Chakraborty, S. Ghosh and N. Ganguly, "Detecting Overlapping Communities in Folksonomies", (2012) June 25-28.

[15] http://en.wikipedia.org/wiki/OverlappingCommunity_structure

[16] http://web.mit.edu/8.334/www/grades/projects/projects10/Hernandez-Lopez Rogelio/ 
International Journal of Grid Distribution Computing Vol. 8, No.4, (2015) 